\title{
Genome-wide analysis of signal peptide functionality in Lactobacillus plantarum WCFS I
}

\author{
Geir Mathiesen*1, Anita Sveen ${ }^{1}$, May Bente Brurberg ${ }^{2}$, Lasse Fredriksen ${ }^{1}$, \\ Lars Axelsson ${ }^{3}$ and Vincent GH Eijsink ${ }^{1}$
}

Address: ${ }^{1}$ Norwegian University of Life Sciences, Center for Molecular Microbiology, Department of Chemistry Biotechnology and Food Science, Chr. M. Falsensvei 1. P.O. Box 5003, N-1432 Ås, Norway, ${ }^{2}$ Norwegian Institute for Agricultural and Environmental Research, Plant Health and Plant Protection Division, Høgskoleveien 7, 1432 Ås, Norway and 3Norfima Mat, Osloveien 1, N-1430 Ås, Norway

Email: Geir Mathiesen* - geir.mathiesen@umb.no; Anita Sveen - anita.sveen@gmail.com; May Bente Brurberg - May.Brurberg@bioforsk.no; Lasse Fredriksen - lasse.fredriksen@umb.no; Lars Axelsson - lars.axelsson@nofima.no; Vincent GH Eijsink - vincent.eijsink@umb.no

* Corresponding author

Published: 10 September 2009

BMC Genomics 2009, 10:425 doi:10.1186/|47|-2/64-10-425
Received: 6 April 2009

Accepted: 10 September 2009

This article is available from: http://www.biomedcentral.com/I47I-2/64/I0/425

(c) 2009 Mathiesen et al; licensee BioMed Central Ltd.

This is an Open Access article distributed under the terms of the Creative Commons Attribution License (http://creativecommons.org/licenses/by/2.0), which permits unrestricted use, distribution, and reproduction in any medium, provided the original work is properly cited.

\begin{abstract}
Background: Lactobacillus plantarum is a normal, potentially probiotic, inhabitant of the human gastrointestinal $(\mathrm{Gl})$ tract. The bacterium has great potential as food-grade cell factory and for in situ delivery of biomolecules. Since protein secretion is important both for probiotic activity and in biotechnological applications, we have carried out a genome-wide experimental study of signal peptide (SP) functionality.

Results: We have constructed a library of 76 Sec-type signal peptides from L. plantarum WCFSI that were predicted to be cleaved by signal peptidase I. SP functionality was studied using staphylococcal nuclease (NucA) as a reporter protein. $82 \%$ of the SPs gave significant extracellular NucA activity. Levels of secreted NucA varied by a dramatic 1800 -fold and this variation was shown not to be the result of different mRNA levels. For the best-performing SPs all produced NucA was detected in the culture supernatant, but the secretion efficiency decreased for the less well performing SPs. Sequence analyses of the SPs and their cognate proteins revealed four properties that correlated positively with SP performance for NucA: high hydrophobicity, the presence of a transmembrane helix predicted by TMHMM, the absence of an anchoring motif in the cognate protein, and the length of the $\mathrm{H}+\mathrm{C}$ domain. Analysis of a subset of SPs with a lactobacillal amylase (AmyA) showed large variation in production levels and secretion efficiencies. Importantly, there was no correlation between SP performance with NucA and the performance with AmyA.

Conclusion: This is the first comprehensive experimental study showing that predicted SPs in the $L$. plantarum genome actually are capable of driving protein secretion. The results reveal considerable variation between the SPs that is at least in part dependent on the protein that is secreted. Several SPs stand out as promising candidates for efficient secretion of heterologous proteins in L. plantarum. The results for NucA provide some hints as to the sequence-based prediction of SP functionality, but the general conclusion is that such prediction is difficult. The vector library generated in this study is based on exchangeable cassettes and provides a powerful tool for rapid experimental screening of SPs.
\end{abstract}




\section{Background}

Lactobacillus plantarum is a Gram-positive lactic acid bacterium (LAB) with a long tradition in food fermentation, and is therefore Generally Regarded As Safe (GRAS status). This microbe is found in many ecological niches including naturally fermented food and decaying plant materials. Furthermore, L. plantarum is a normal inhabitant of the human gastrointestinal (GI) tract [1]. The complete genome sequence of L plantarum WCFS1 has been determined [2], and tools for genetic engineering are available [3-7]. L. plantarum is adapted to survive in the harsh conditions of the GI-tract, as has been illustrated by recent genome-wide gene expression studies of the response of the bacterium to (mouse) GI-tract conditions [8,9]. Both the potential probiotic effects of $L$. plantarum and the high survival rate during the passage of the GI-tract make this bacterium a promising candidate as a vehicle for in situ delivery of therapeutically interesting proteins [10]. The general potential of LAB as in situ delivery vehicles for biomolecules is well recognized. For example, a recent phase I trial study has indicated that Crohn's disease patients benefit from treatment with a genetically modified Lactococcus lactis secreting human interleukin 10 [11]. Promising results have been obtained with LAB that secrete or anchor antigens to the cell (recently reviewed by Wells and Mercenier [12]; see also [13]).

Bacteria use several pathways for protein export to the membrane, the cell wall or the medium [14]. Many proteins follow the Sec-dependent pathway and are synthesized as precursors with an N-terminal signal peptide that directs the protein to the Sec translocation machinery. In the case of Sec-dependent secreted proteins, the signal peptide is cleaved off during or shortly after the translocation [15-17]. The genome of L. plantarum WCFS1 codes for more than 200 proteins that contain an N-terminal signal peptide. About 100 of these proteins contain a potential signal peptidase I cleavage site, and are thus likely to be secreted to the culture medium or anchored to the cell wall $[2,18]$. For the large majority of the proteins whose secretion is directed by these signal peptides experimental data showing functional properties are lacking. Using bioinformatics, some of the proteins were predicted to be enzymes or to be involved in adherence to host components [18].

The possibility to secrete heterologous proteins in $L$. plantarum or other LAB has been addressed in several studies [5,19-25]. So far, engineered secretion in L. plantarum has mostly been based on the use of heterologous signal peptides. The most widely exploited heterologous signal peptides are those from the L. lactis Usp45 protein [26$28]$, the Streptococcus pyogenes M6 protein $[5,27,29]$, and the $L$. brevis S-layer protein $[20,24]$, as well as signal peptides from different microbial amylases $[4,30]$. When aiming for the construction of genetically engineered $L$. plantarum strains for human consumption, there is a need for the use of homologous signal peptides since this limits the use of foreign DNA and since homologous signal peptides may lead to more efficient secretion. One key problem in selecting suitable signal peptides is the difficulty in predicting their efficiency on the basis of their sequence only (see below).

In this study we present the first genome-wide experimental analysis of the functionality of SPs from lactic acid bacteria. We have conducted a functional analysis of 76 of the 93 signal peptides from $L$. plantarum WCFS1 that were predicted by Kleerebezem et al. [2] to be processed by signal peptidase I. Seventeen of the 93 SPs were discarded from the study, primarily because the prediction of the cleavage site was ambiguous. To study the functionality of the signal peptides, they were used to direct secretion of a nuclease (NucA) from Staphylococcus aureus and, for a subset, an amylase (AmyA) from Lactobacillus amylovorus. This screening revealed large variation in signal peptide functionality and led to identification of some homologous signal peptides that yielded high secretion levels in $L$. plantarum. Although we generally found little correlation between signal peptide sequence properties and secretion results, our genome-wide data do suggest some criteria that may be used to increase the likelihood of selecting signal peptides (SPs) that yield efficient secretion of heterologous proteins.

\section{Results}

\section{Library construction}

Kleerebezem et al. [2] identified 93 proteins with putative signal peptidase I cleavage sites in the genome of $L$. plantarum WCFS1. In this study we ran all the 93 protein sequences through the web-based SignalP 3.0 program, using both the neural network (NN) and hidden Markov model (HMM) algorithms to predict putative cleavage sites [31]. The two algorithms yielded the same conclusions for 78 SPs and these were selected for further studies. Two of the 78 sequences were omitted from the SP library, one (Lp_0374) because it's coding DNA contains a SalI site and one because of cloning problems (Lp_0946). An overview of the 76 remaining SPs and two additional heterologous SPs (M6 \& Usp45) included in the library is presented in additional file 1.

The length of the selected SPs varies from 24 (several proteins) to 57 (Lp_2796) residues. The large majority of the SPs (61 of 76) had lengths between 24 and 36 residues, and only one sequence was predicted to be longer than 50 residues (Lp_2796). Analyses of bacterial SPs have shown predominance for alanine at positions $-3,-1$ and +1 relative to the cleavage site $[17,32,33]$. Seventeen of the 76 selected sequences have the consensus Ala-X-Ala $\downarrow$ Ala cleavage site, whereas 47, 74 and 33 of the SPs contain an Ala in the $-3,-1$ and +1 positions, respectively. At position 
$-2,15$ different residues are present, both small non-polar, polar and charged. The most dominant residue at the -2 position is glutamine which is present in 17 of the sequences. Those SPs that do not have Ala in -3 have small, non-polar residues at this position. In the +1 position Ala is most often replaced by Asp ( $25 \mathrm{SPs}$ ). Weblogos [34] for the predicted cleavage sites of all 76 SPs and some subgroups of SPs are presented in additional file 2.

The SP library was constructed by fusing SPs translationally to the start codon of the sppA gene downstream of its native inducible $\mathrm{P}_{\mathrm{sppA}}$ promoter using an $\mathrm{NdeI}$ restriction site, as described in Methods. At the C-terminal end of the SPs, two amino acids downstream of the predicted cleavage site were retained from the original protein. Because the SPs were fused to the NucA reporter protein by a 6 nucleotide linker creating a unique SalI restriction site, every construct had a valine followed by an aspartic acid residue in positions +3 and +4 relative to the cleavage site. The staphylococcal NucA was selected as a reporter protein because of its stability, small size, easily measurable extracellular activity and because it has previously been successfully used as a reporter protein for secretion in lactic acid bacteria [26,35].

\section{Secretion capacity of the SP library}

To measure the secretion capacity of the SPs in the library, induced cells were harvested at $\mathrm{OD}_{600} \sim 1.7$ (late logarithmic phase) and nuclease activity was measured in cell free supernatants. Figure 1 shows that there is large variation in the secretion capacity among the SPs. For 14 of the SPs extracellular nuclease activity was not significantly higher

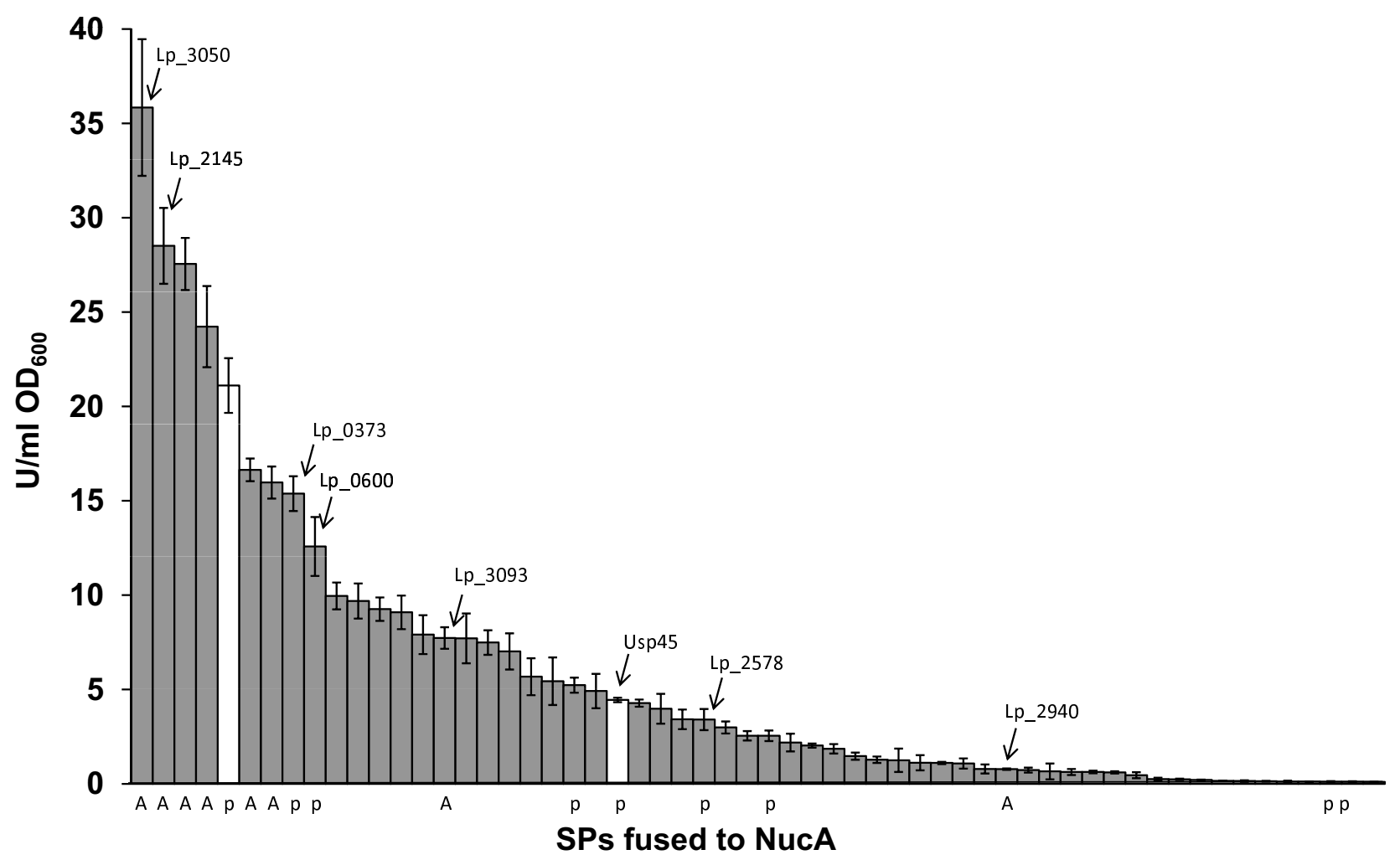

\section{Figure I}

Nuclease activities in supernatants of $L$. plantarum WCFSI harbouring plasmids with different signal peptides (SPs). Signal peptides whose functionality in NucA secretion was tested in more detail by western blotting (see text) are marked with an arrow and are labeled with the corresponding gene code. The white bars represent the two heterologous SPs that were included in this study. Signal peptides that were also tested with the amylase reporter are marked with an A below the X-axis. Signal peptides whose functionality for NucA and AmyA has been addressed in a previous study [19] are marked with a $p$ below the $X$-axis. Enzyme activities are expressed in Units per $\mathrm{ml}$ divided by $\mathrm{OD}_{600}$. All results are the mean of three independent experiments; the error bars indicate the standard deviation. Only the 58 SPs that led to an extracellular nuclease activity equal to or higher than $0.1 \mathrm{U} / \mathrm{ml} \mathrm{OD}_{600}$ are shown. Extracellular nuclease activities for the complete SP library are given in additional file I. 
than the activity found for the construct with no signal peptide $\left(0.02 \mathrm{U} / \mathrm{ml} . \mathrm{OD}_{600} ; \mathrm{p}<0.05\right.$ by t-test; data in additional file 1). The other constructs yielded a continuum of activity levels spanning from close to $0.02 \mathrm{U} / \mathrm{ml} \mathrm{OD}_{600}$ to the almost 1800 -fold higher value of $35.8 \mathrm{U} / \mathrm{ml} \mathrm{OD}_{600}$ obtained for the best signal peptide, Lp_3050. We also compared the secretion capacity of the SPs in the library to the secretion capacity of commonly used heterologous SPs derived from the Usp45 (L. lactis) and the M6 (S. pyogenes) proteins. Both heterologous SPs yielded secretion of NucA and the SP from M6 was among the better performing SPs (Figure 1).

To check whether the variation in apparent secretion capacities was caused by differing transcription levels, we determined transcript levels in cultures from a subset of the cloned constructs using real-time PCR analyses. The results, depicted in Figure 2, show that there were no significant differences in transcript levels.

NucA secretion efficiency of selected SPs in L. plantarum To investigate secretion efficiencies (i.e. the fraction of produced protein that is secreted), comparable amounts of cell and supernatant fractions were analyzed by Western blot experiments using a polyclonal antiserum against NucA. We selected eight SP constructs from the library covering a wide range of secretion capacities (Figure 1). Processed NucA could be detected in the supernatant of all selected clones (Figure 3), but not in the supernatant fraction of the control L. plantarum harboring the pNuc-

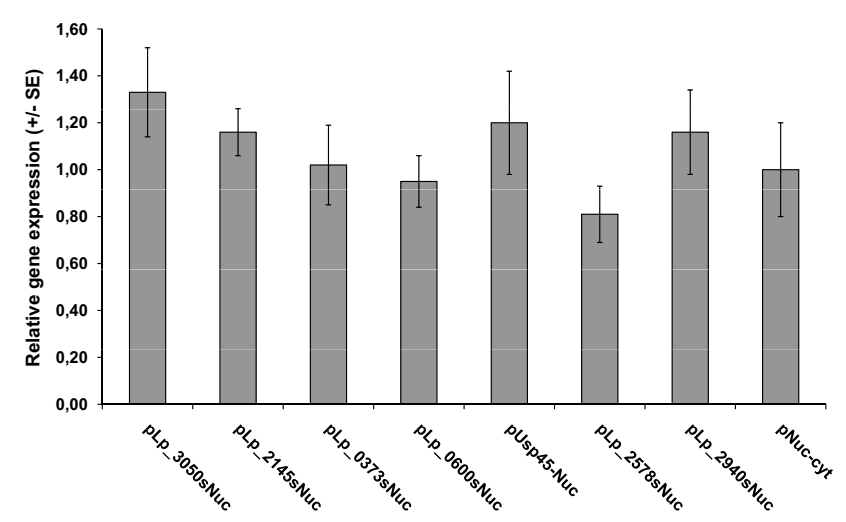

\section{Figure 2}

Expression of NucA in recombinant L. plantarum strains as estimated using real-time PCR analyses.

The bars show expression ratios for nucA, calculated by comparing transcript levels in $L$. plantarum strains harbouring various constructs with the level in L. plantarum/pLp_3093sNuc, which was arbitrarily chosen as a control. The data are the mean of two independent experiments; standard errors (SE) are indicated. All $\mathrm{C} t$ values were normalized against gyrA using the REST-program.
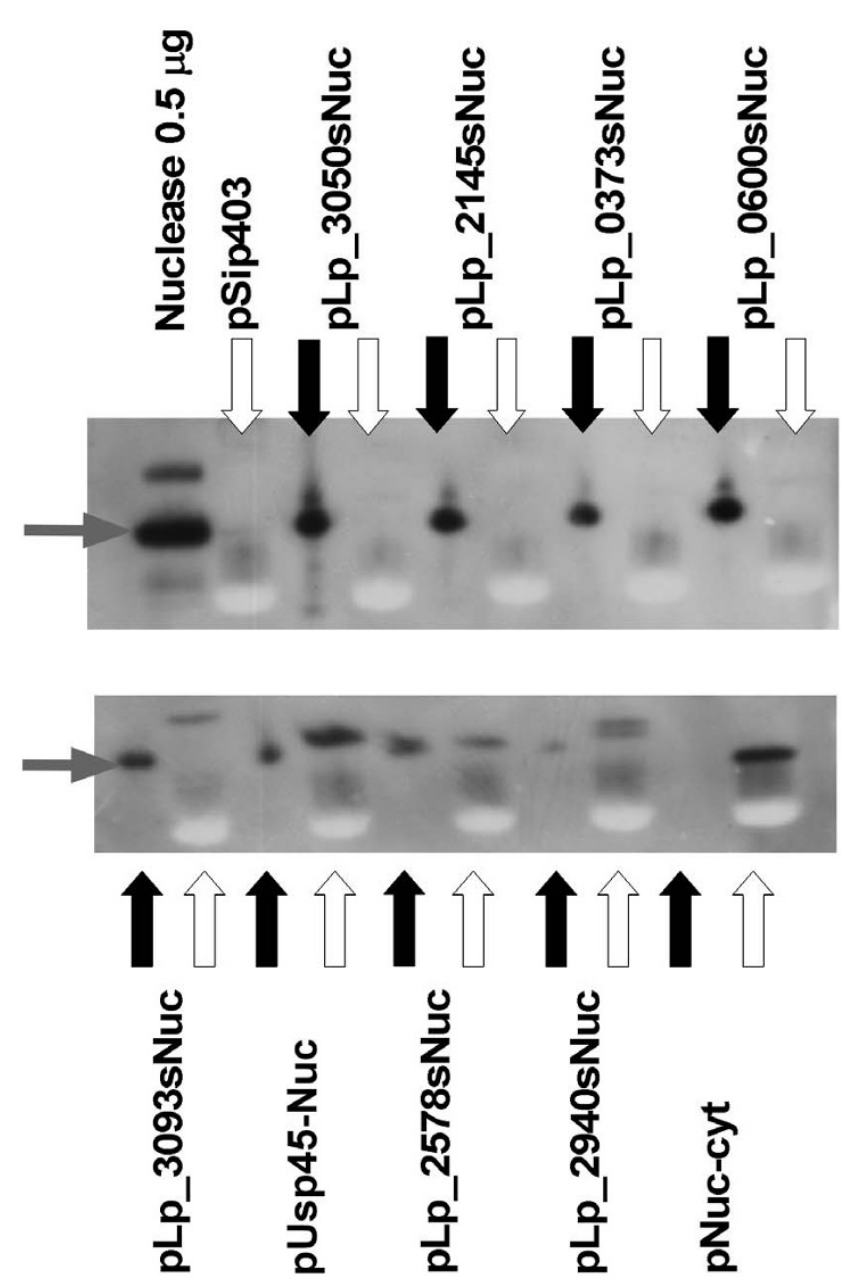

Figure 3

Western blots for analysis of secretion efficiency. White and black arrows indicate the cell lysate and supernatants fractions, respectively. Grey arrows indicate mature NucA. In addition to the results for eight constructs with signal peptides, the gels shows results for a construct driving cytoplasmic production of NucA (pNuc-cyt), a construct without the nucA gene (PSIP403, a construct for intracellular expression of gusA; [3]; only the cell lysate is shown for this construct) and a sample of pure mature NucA. For all the culture-derived samples, the sample size corresponded to 16 $\mu \mathrm{l}$ of the original culture harvested at an $\mathrm{OD}_{600}$ of approximately I.7.

cyt construct lacking a signal peptide. Both the fact that processing occurred and the lack of extracellular NucA in the pNuc-cyt control show that extracellular NucA detected in the transformants is not due to cell lysis. The amount of NucA detected in the supernatants by Western blotting generally corresponded well with the measured extracellular NucA activities shown in Figure 1. 
Figure 3 shows that the secretion efficiency was close to $100 \%$ for the four SPs that yielded the highest extracellular activities, while secretion efficiencies were lower for the rest of the constructs. In these latter cases unprocessed NucA accumulated intracellularly. Using a sample of pure NucA as a standard, the amount of secreted NucA obtained with the pLp_3050sNuc plasmid was estimated to be in the range of $5-10 \mathrm{mg} / \mathrm{l}$ culture.

\section{Secretion of L. amylovorus $\alpha$-amylase (AmyA)}

In order to test the SPs' general usefulness to direct secretion, the six SPs leading to highest NucA secretion were also used to direct secretion of the $49 \mathrm{kDa}$-terminal fragment of the $\alpha$-amylase (AmyA) from L. amylovorus NRRL B-4549 [36,37]. For comparison, two additional SPs that led to lower extracellular NucA levels (Lp_3093 \& Lp_2940) were included. The performance of the new constructs was analyzed by activity measurements (Table 1) and SDS-PAGE (additional file 3). All SP-containing constructs led to extracellular amylase activity but secretion efficiencies were well below $100 \%$ in all cases. The latter contrasts with the observations made for NucA, where secretion efficiencies for the better constructs approached 100\% (Figure 3). The total AmyA activity (intra-plus extracellular) varied greatly among the constructs and generally the constructs yielding highest total activity displayed the lowest secretion efficiency. In line with previous observations [19], Table 1 shows that there generally is little correlation between the performance of an SP in NucA secretion and its performance in AmyA secretion. The trend in the data in Table 1 seems to be that SPs leading to intermediate secretion levels of NucA are among the best performers for AmyA, both with respect to the total level of extracellular activity and secretion efficiency.

Using silver stained SDS-PAGE gels (additional file 3), the secreted amylase could be detected in non-concentrated culture media and the relative intensities of the bands correlated well with the relative levels of enzyme activities. In the case of the pLp_2940sAmy construct, which leads to high total amylase levels, cell lysis was observed. Scanning electron microscopy showed that induced pLp_2940sAmy containing cells had an elongated shape that differed drastically from the shape of non-induced pLp_2940sAmy containing cells and induced cells containing other secretion constructs such as pLp_0297sAmy (Figure 4).

\section{Correlations between SP properties and secretion capacity for NucA}

To search for a connection between SP sequence properties and secretion capacity, we carried out a series of calculations based on the SP sequences and compared the results with the NucA activity data. Tables 2 and 3 present the results of two types of group-wise analyses. In one analysis, the ten best performing SPs (1-10) were compared with SPs without significant extracellular activity

Table I: Secretion efficiency and $\alpha$-amylase activity in recombinant $L$. plantarum WCFSI harbouring various constructs ${ }^{a}$.

\begin{tabular}{|c|c|c|c|c|}
\hline Plasmids & $\begin{array}{l}\text { Cell fractions } \\
\left(10^{2} \mathrm{mU} \mathrm{ml}^{-1} O \mathrm{D}_{600^{-1}}\right)\end{array}$ & $\begin{array}{l}\text { Culture supernatants } \\
\left(10^{2} \mathrm{mU} \mathrm{ml^{-1 }} O D_{600^{-1}}\right)\end{array}$ & $\begin{array}{l}\text { Secretion efficiency } \\
(\%)^{c}\end{array}$ & Ranking number after NucA activity \\
\hline pLp_3050sAmy & $7.2 \pm 0.6$ & $0.57 \pm 0.04$ & 7 & I \\
\hline pLp_2I45sAmy & $28.0 \pm 3.6$ & $0.92 \pm 0.04$ & 3 & 2 \\
\hline pLp_3189sAmy & $8.7 \pm 1.0$ & $0.92 \pm 0.03$ & 10 & 3 \\
\hline PLp_3077sAmy & $29.9 \pm 5.1$ & $0.43 \pm 0.04$ & 1 & 4 \\
\hline pM6sAmy & $53 \pm 10$ & $0.40 \pm 0.17$ & 0.7 & 5 \\
\hline pLp_0297sAmy & $7.2 \pm 2.6$ & $3.4 \pm 0.34$ & 32 & 6 \\
\hline pLp_31I7sAmy & $6.8 \pm 0.8$ & $0.45 \pm 0.1$ & 6 & 7 \\
\hline pLp_0373sAmy & $5.3 \pm 0.1$ & $2.3 \pm 0.1$ & 30 & 8 \\
\hline PLp_0600sAmyb & $1.8 \pm 0.4$ & $1.7 \pm 0.2$ & 49 & 9 \\
\hline pLp_3093sAmy & $40.7 \pm 2.0$ & $0.30 \pm 0.04$ & 1 & 15 \\
\hline pLp_2958sAmy & $2.8 \pm 0.8$ & $1.5 \pm 0.1$ & 35 & 21 \\
\hline pUsp45sAmyb & $29 \pm 6$ & $0.37 \pm 0.14$ & 1 & 23 \\
\hline pLp_2578sAmyb & $2.4 \pm 0.5$ & $0.57 \pm 0.2$ & 19 & 27 \\
\hline pLp_2588sAmyb & $2.0 \pm 0.2$ & $2.1 \pm 0.1$ & 51 & 30 \\
\hline pLp_2940sAmy & $23.8 \pm 6.4$ & $(3.2 \pm 0.3)^{\mathrm{d}}$ & n.d.d & 41 \\
\hline pLp_3127sAmy & $28 \pm 2$ & $0.05 \pm 0.01$ & 0.2 & 56 \\
\hline pLp_1447sAmy & $1.5 \pm 0.2$ & $0.39 \pm 0.08$ & 21 & 57 \\
\hline pAmy-cyt & $12.6 \pm 4.6$ & $0.05 \pm 0.04$ & 0 & \\
\hline
\end{tabular}

a Plasmids encoding SPs are ranked according to their capacity to secrete NucA, as shown in Figure I (best SP first). All results are the mean value of three biological replicates.

b Data from Mathiesen et al [19]. Note that the selection of SPs in this previous study was more or less random and not based on the genome-wide overview of SP functionality that is presented here.

c Based on the assumption that all intracellular, non-processed AmyA is equally active as secreted AmyA.

dApparent secretion is partly due to cell lysis, meaning that the secretion efficiency could not be calculated. See text for details. 

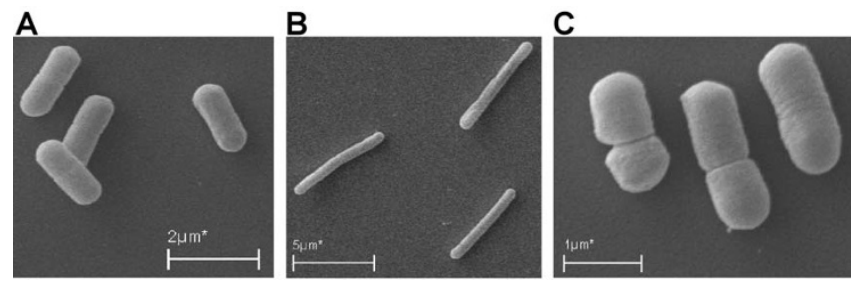

Figure 4

Scanning electron microscopy images of recombinant $L$. plantarum WCFS $I$, harvested at $\sim O D_{600} I .7$. A, non-induced cells harboring PLP_2940sAmy; B, induced cells harboring PLP_2940sAmy; C, induced cells harboring pLp_0297sAmy.

(65-78). In the other analysis, the best performing half of the SPs (1-39) was compared with the least performing half (40-78). For each group mean values for the different properties were calculated and these values were then compared. For some of these analyses the SPs were divided into domains [17]. The $\mathrm{N}$-domain was defined to span from the $\mathrm{N}$-terminal methionine to the last positively charged residue in the N-terminal part of the SP, and had an average length of 8.3 residues. The rest of the $\mathrm{SP}$ is referred to as $\mathrm{H}+\mathrm{C}$ domain, consisting of the hydrophobic H-region following the $\mathrm{N}$-domain, followed by the C-domain that ends at the predicted cleavage site for the signal peptidase.

Comparing the groups did not yield significant correlations between measured extracellular NucA activities and the following SP properties: isoelectric point of the complete SP, length of the SP, net charge or length of the $\mathrm{N}$ domain, net charge/length of the $\mathrm{N}$-domain, and the $\mathrm{D}$ value provided by SignalP (see additional file 1 for raw data). However, the data showed that measured NucA activities were significantly $(\mathrm{p}<0.05)$ correlated to SP properties as follows: (1) a positive correlation with SP hydrophobicity, found in both comparisons (1-10 vs 6578 and $1-39$ vs $40-78)$; (2) a positive correlation with the length of the $\mathrm{H}+\mathrm{C}$ domain. A control analysis using only SPs that gave significant extracellular activity (1-32 versus 33-64) yielded the same correlations (results not shown).

Analysis of the sequence of the cleavage sites did not show any clear trends. In fact, the data did not suggest that the presence of the consensus sequence Ala-X-Ala $\downarrow$ Ala is particularly favourable. Both the A-X-A motif in front of the cleavage site and the $\mathrm{A}$ at position +1 were more abundant in the least performing half of the SPs. Only six of the 39 best performing SPs had the A-X-A $\downarrow$ A consensus sequence.

Previous studies have shown that SPs adopt $\alpha$-helical conformations in interfacial environments such as cell membranes [38,39]. All 76 SPs as well as M6 and Usp45 were run through a web-based transmembrane helical prediction program, TMHMM Server v. 2.0 [40]. The prediction showed that 62 of the 78 SPs were predicted to adopt a transmembrane helix (TMH) structure. Interestingly, 97\% of the 39 best performing SPs were predicted to contain a $\mathrm{TMH}$, while this was the case for only $62 \%$ of the 39 worst performing SPs (Table 3; raw data see additional file 1). The observed secretion capacities showed no correlation with the length of the predicted TMH nor with the position of the predicted helix start (see additional file 1 for raw data). TMHMM also predicts the presence of SPs. Nine of the 78 SPs were not recognized as SPs by TMHMM and eight of these were all in the least performing half of the 78 tested SPs (additional file 1).

Interestingly, there also seems to be a weak correlation between the ability of an SP to drive secretion of NucA and the presence of motifs that keep the original cognate protein attached to the cell surface (LysM, LPxTG and Cterminal membrane anchoring motifs as predicted by

Table 2: Correlations between SP properties and measured extracellular NucA activities. ${ }^{a}$

\begin{tabular}{|c|c|c|c|c|c|c|c|}
\hline $\begin{array}{l}\text { Ranked after } \\
\text { NucA activityb }\end{array}$ & $\begin{array}{l}\text { Lenght } \\
\text { of SPs }\end{array}$ & pl of SPs & Hydro-phobicityc & $\begin{array}{l}\text { Length of } \\
\text { N-domain }\end{array}$ & $\begin{array}{l}\text { Net charge of } \\
\text { the N-domain }\end{array}$ & $\begin{array}{l}\text { Charge/length of } \\
\text { the N-domain }\end{array}$ & $\begin{array}{c}\text { Length of H-plus } \\
\text { C-domains }\end{array}$ \\
\hline $1-10$ & 34.5 & 9.8 & 2.98 & 7.2 & 3.1 & 0.5 & 27 \\
\hline $65-78$ & 32.8 & 10.1 & 2.31 & 9.9 & 3.0 & 0.4 & 23 \\
\hline $1-39$ & 31.6 & 10.3 & 2.77 & 7.4 & 2.9 & 0.5 & 24 \\
\hline $40-78$ & 31.1 & 10.1 & 2.26 & 9.1 & 3.0 & 0.4 & 22 \\
\hline $1-78$ & 31.3 & 10.2 & 2.52 & 8.3 & 3.0 & 0.4 & 23 \\
\hline
\end{tabular}

aAverage values for different properties were calculated for SPs grouped by NucA activity.

bSubgroups of the 78 SPs were made as indicated, with SPs being numbered by their ability to produce extracellular NucA activity, with SP number I having the highest activity; see text for further details.

c The hydrophobicity of the SPs was estimated using the ProtScale program [66] using the Kyte \& Doolittle scale [67] on the ExPASy Server http:// www.expasy.ch/tools/protscale.html. The numbers indicate the maximum hydrophobicity value, using a sliding window size of seven.

$\mathrm{d}$ See text for definition of the domains. Nb. We verified that the length of the $\mathrm{H}+\mathrm{C}$ domain is not correlated to the maximum hydrophobicity defined in footnote $c$ (results not shown). 
Boekhorst et al. [18]; see additional file 1). Of the 41 proteins in the library harbouring one of these motifs, most have SPs that led to low secretion capacity for NucA (Table 4). $64 \%$ of the proteins belonging to the 39 worst performing SPs were predicted to contain a cell-wall anchoring motif, while this was the case for only $41 \%$ of the 39 proteins with the best performing SPs (Table 4 and additional file 1).

\section{Discussion}

We present a comprehensive study of putative SPs in the genome of L. plantarum WCFS1 for which SignalP predicted a unique cleavage site for signal peptidase I. The results provide genome-wide insight into SP functionality, new tools (vectors) for secretion of proteins using homologous SPs, and increased insight into the predictability of SP functionality on the basis of sequence only.

$82 \%(\mathrm{p}<0.05)$ of the 76 tested SPs led to secretion of NucA. While this result may be taken to confirm that the $62 \mathrm{~L}$. plantarum proteins containing these SPs indeed are secreted, it does not imply that the remaining 14 SPs do not function at all and that their cognate proteins are not secreted. SP functionality depends on which protein is being secreted $[41,42]$, meaning that SPs that do not work for NucA may function when coupled to another protein (and, in principle, vice versa). Furthermore, in some cases prediction of the signal peptidase cleavage site may have been wrong, despite the unanimous prediction by the two Signal P algorithms (see also below). Indeed, comparison of the sequences of some of the seemingly non-functional SPs (see additional file 1) with what is known about cleavage site sequences (illustrated by the sequence logos in additional file 2) show that alternative cleavage sites are possible in some of these SPs. The detected levels of extracellular NucA varied by three orders of magnitude. Since the only difference between the constructs is the SP, the large differences in secretion capacity are due to variation in the SP, directly or indirectly. To try to unravel the causes of these variations we set up additional experiments and looked closer into the properties of the SPs.

Real-time PCR studies of cultures containing different constructs did not reveal significant differences in mRNA levels. This indicates that the large variation in secretion capacity observed for these constructs is not due to differences in transcription levels. This is an expected result, since the constructs contain identical transcription initiation and termination signals. Thus, the variation in secretion capacities must be governed by (inter-related) posttranscriptional factors such as secondary structure of mRNA, codon usage and translation efficiency, the interaction between the precursor protein and the translocation machinery, the efficiency of the signal peptidase for the SP in question, the rate of (non-desirable) intracellu- lar and (desirable) extracellular folding, and possible interactions between the secreted protein and the bacterial cell wall [41,43-45].

Although the Western blot of Figure 3 provides only limited quantitative insight, the data do suggest that all $L$. plantarum transformants produced approximately equal amounts of NucA, meaning that all transformants experienced approximately equal "protein loads". The data show a (rough) correlation between translocation efficiency and the levels of secreted protein (Figure 3). One possible cause of variation in secretion efficiency is variation in the efficiency of SP processing. However, in their genome-wide study of B. subtilis SPs Brockmeier et al. [42] showed that the rate of precursor processing had limited effects on levels of extracellular reporter protein. Assuming a similar situation in L. plantarum, differences in the efficiency of the translocation process itself remain as the main cause of the variation in extracellular NucA levels.

Mutagenesis studies have confirmed that secretion levels in Gram-positive bacteria are not only affected by variation in the SPs [46-48] but also by variation in the N-terminal part of the mature protein [21,35]. Le Loir et al. [21] showed that negative charge in the $\mathrm{N}$-terminal part of the secreted protein was beneficial for secretion. The NucA variants in the present study varied only with respect to residues +1 and +2 and we did not observe correlations between the character of these residues and secretion performance of the SP. The very efficient Lp_3050 sequence has a basic residue (Lys) at position +2 which is unexpected on the basis of the conclusions drawn by Le Loir et al. [21]. Taking into account the above considerations, it is likely that the variation in the secretion of NucA observed in this study is caused by the variation of the SP only and its effect on the interaction between the precursor and the translocation machinery.

The translocation process is a complex process which involves many interactions that are affected by the characteristics of both the SP and the protein. It is conceivable, that SPs are evolutionary adapted to their cognate protein to ensure efficient and controlled secretion. The importance of the protein part is clearly shown in both the present study and a previous genome-wide study on SPs from $B$. subtilis [42], which show that the efficiency of many SPs depends on the reporter protein. Thus, high secretion efficiency requires an optimal combination between the SP and the target protein. Recent studies suggest that SP function may be much more complex than previously thought, and may direct surface proteins to different subcellular locations [49-51]. Clearly, such underlying complexities in SP functionality, will weaken correlations between SP sequence properties and secretion levels. 
Several studies have shown that changes in hydrophobicity of the H-domain can affect the secretion capacity $[47,52,53]$ and this is indeed one of the correlations that we discovered in the present genome-wide study. However, in a study of 148 SPs from B. subtilis [42] no such correlation was found. In the present study, we also identified a clear correlation between a predicted transmembrane helix by the programme TMHMM and high secretion capacity. On the basis of our results, running TMHMM seems one of the best ways to select SPs that are likely to perform well, and this analysis should thus be performed next to SignalP. In addition, the length of the $\mathrm{H}+\mathrm{C}$ domain should also be taken into account when selecting an SP. It is interesting to note that SPs from proteins that are thought to be anchored to the cell wall tend to perform less well than other SPs. It is conceivable that these proteins do not require high translocation efficiencies, since they are not meant to be actively secreted to the surrounding media and therefore may be produced at lower levels than released proteins.

In this study, we have based the prediction of signal peptides on the original analysis of the L. plantarum genome as described by Kleerebezem et al [2] and we have used SignalP 3.0 to check and predict the cleavage sites. Clearly, the annotation of the L. plantarum genome will evolve as bioinformatic tools evolve and today's annotation, e.g. with respect to the subcellular localization of proteins, will differ from the one published in 2003. The most accurate prediction of extracellular protein localization in $L$. plantarum WCFS1 is found in the Secretome database [18]http://www.cmbi.ru.nl/secretome. Another prediction tool is the newly developed Locate P [54] that combines existing predictors and produces genome-wide predictions for the subcellular locations of bacterial proteins in a fully automated manner. Predictions based on both methods/databases for the 78 proteins relevant for this study are included in additional file 1 and show several differences. For example, all but one (Lp_1524) of the selected SPs are predicted to be cleaved by SPaseI in the Secretome database, whereas Locate $P$ predicts such cleavage only for 63 of the SPs. The present set with experimental data may be used to evaluate prediction quality and, hopefully, to improve prediction methods. Our data show that the SPs of several proteins predicted to be N-terminally anchored by LocateP lead to efficient secretion of NucA, meaning that they are cleaved by SPaseI as predicted by SignalP and according to the prediction in the Secretome database. Likewise, several proteins predicted to be multi-membrane proteins according to Locate $\mathrm{P}$ contain SPs that are quite efficient for NucA secretion.

To test the general performance of the SPs we replaced NucA with AmyA in selected constructs. When produced at levels applied in this study, AmyA seems to be difficult to handle for $L$. plantarum. Secretion efficiencies were below, often far below, 100\% for all constructs. Table 1 shows that the AmyA constructs lead to highly variable overall production levels, creating a complicating variable that was less prominent in the studies with NucA. Previous studies have shown that overexpressed amylase can be difficult to handle for $B$. subtilis and induce stress reactions $[55,56]$. Table 1 also shows that high production levels of AmyA correlate with low secretion efficiencies, suggesting that the translocation machinery is overloaded. In addition to slow or blocked translocation, secretion stress may cause intracellular or extracellular proteolytic degradation $[41,56]$. Proteolytic degradation was not analyzed because of the lack of a suitable antibody for AmyA. The stress caused by AmyA expression is illustrated by cells harbouring the pLp_2940sAmy construct that leads to high levels of AmyA production. These cells showed impaired growth (data not shown), cell lysis and a change in morphology (Figure 4). Lp_2940 did not perform very well for NucA (rank 41) and it does not have the properties that are typical for SPs that work well with NucA (see additional file 1). It is possible that the combination of a high production level with an unfavourable SP stressed the cells to the extent that lysis occurred. All in all, our observations with AmyA indicate that this protein is not a suitable reporter to search for characteristics in SPsequences that correlate to secretion capacity.

\section{Conclusion}

The present study shows that at least $82 \%$ of the tested putative signal peptidase I-dependent SPs in the genome of $L$. plantarum WCFS1 indeed functions as a signal for secretion. The results reveal considerable variation in SP performance that is at least in part dependent on the protein that is secreted. We identified correlations between SP sequence and SP performance which may be used for preselecting promising SPs, but the general conclusion is that prediction of SP performance is difficult. The lack of predictability suggests that sequence differences between SPs at least in part relate to other (potential) aspects of SP functionality, such as spatial and temporal regulation of protein production and secretion. As it stands, secreting a protein of interest at the highest possible levels in $L$. plantarum will require experimental screening of SPs. The library constructed in this study provides an easy to use tool for rapid experimental screening since it is based on exchangeable cassettes.

\section{Methods}

\section{Bacterial strains and growth conditions}

Escherichia coli TOP10 (Invitrogen, Carlsbad, CA, USA) cells were grown in BHI broth (Oxoid Ltd., Hampshire, England) at $37^{\circ} \mathrm{C}$ with shaking. L. plantarum WCFS1 [2] was grown in MRS broth (Oxoid) at $30^{\circ} \mathrm{C}$ without agitation. Solid media were prepared by addition of $1.5 \%(\mathrm{w} /$ 
v) agar. Antibiotics were added as follows: for E. coli, kanamycin $100 \mu \mathrm{g} / \mathrm{ml}$ and erythromycin, $200 \mu \mathrm{g} / \mathrm{ml}$; for $L$. plantarum, erythromycin $5 \mu \mathrm{g} / \mathrm{ml}$.

\section{Standard genetic techniques and transformation}

Primers used in this study were purchased from Operon Biotechnologies GmbH (Cologne, Germany) and are listed in additional file 4. Chromosomal DNA from $L$. plantarum was isolated using the E.N.Z.A Bacterial DNA kit (Omega Bio-Tek. Inc. Doraville, GA) by following the protocol provided by the manufacturer. Mutanolysin, 15 $\mathrm{U} / \mathrm{ml}$, was added to the cell lysis step in this protocol. All signal sequences were amplified from chromosomal DNA using Phusion polymerase (New England Biolabs, Inc., Ipswich, MA). The PCR fragments were isolated from a 3.5\% NuSieve GTG agarose gel (Cambrex Bio Science Rockland, Inc. Maine) using the NucleoSpin Extract II kit (Macherey-Nagel GmbH \& Co, Düren, Germany) and subsequently sub-cloned into PCR-Blunt II TOPO vector (Invitrogen, Carlsbad, CA) following the protocol from the manufacturer. The sequences of all PCR-generated inserts were confirmed by DNA sequencing.

Chemically competent E. coli TOP10 cells were transformed by following the protocol of the manufacturer and lactobacilli were transformed according to Aukrust et al. [57].

\section{Cloning strategy}

The gene expression system used in this study is based on the modular pSIP-vectors that contain a peptide-pheromone inducible expression system for use in Lactobacillus $[3,58]$. This system has recently been modified to allow secretion of proteins by adding a "signal peptide cassette" [19]. In this system the N-terminal end of the desired SP is translationally fused to the inducible Sakacin P promoter $\left(\mathrm{P}_{s p p A}\right)$ in a modified version of plasmid pSIP401, using a NdeI restriction site at the start codon. The C-terminal end of the SP followed by an additional two amino acids downstream of the predicted cleavage site is fused in-frame to the reporter protein via a Val-Asp linker that yields a unique SalI site at the DNA level. This SP-cassette module permits easy exchange of the SPs by using NdeISalI restriction cloning.

SP sequences were amplified using PCR with primer pairs (named after the gene number in the L. plantarum genome, see additional file 4) harbouring NdeI or SalI sites and the resulting PCR fragments were cloned into PCR-Blunt II TOPO vector (Invitrogen). The SP-containing fragment was excised from the resulting plasmid by NdeI-SalI restriction digesting and ligated into the $6.1 \mathrm{~kb}$ NdeI-SalI fragment of pUsp45-Nuc[19], yielding constructs for secretion of NucA. Some selected SPs were also ligated into the $6.9 \mathrm{~kb}$ NdeI-SalI fragment of pUsp45-Amy [19], yielding constructs for secretion of AmyA. All SPs used for making constructs are listed in additional file 1. As controls we used plasmids pNuc-cyt and pAmy-cyt [19] which direct production of non-secreted NucA and AmyA, respectively.

\section{Nuclease and amylase assays}

Freshly inoculated cultures of L. plantarum WCFS1 harbouring a pSIP-derived plasmid (MRS, $30^{\circ} \mathrm{C}, 5 \mu \mathrm{g} / \mathrm{ml}$ erythromycin) were induced at an $\mathrm{OD}_{600}$ of 0.3 by adding the inducing peptide for sakacin P production [59] to a final concentration of $25 \mathrm{ng} / \mathrm{ml}$. Cells were harvested in late-logarithmic phase at an $\mathrm{OD}_{600}$ of approximately 1.7. NucA activity in the supernatants was measured using the procedure described by Heins et al. [60]. The assay is based on release of acid soluble oligonucleotides from Calf Thymus DNA (Worthington, Lakewood, NJ, USA). One unit of nuclease activity corresponds to an activity generating an $\Delta \mathrm{OD}_{260}$ of 1 per min under the conditions of the assay.

Amylase activity in the supernatant was measured directly using the Phadebas kit (Magle Life Sciences, Lund, Sweden) according to the manufacturer's procedure, with the following modifications: the sample volume was 0.5 or $0.05 \mathrm{ml}$ and the reactions were conducted at $50^{\circ} \mathrm{C}$. To measure intracellular amylase activity, the cells were harvested, washed once with $\mathrm{dH}_{2} \mathrm{O}$, and resuspended in onefifteenth of the original volume. The cells were disrupted by glass beads (Sigma) using FastPrep-24 instrument (MP Biomedicals, Solon, $\mathrm{OH}$ ) to obtain crude protein extracts. Amylase activities were calculated using a standard curve made with $\alpha$-amylase purchased from Sigma (product number A-6380), using the Unit definition provided by Sigma.

\section{SDS-PAGE}

Proteins in cell-free supernatants and intracellular proteins were visualized by running 10\% NuPAGE Novex Bis-

Table 3: Correlation between predicted transmembrane helices (TMH) in the SPs and measured extracellular NucA activities.

\begin{tabular}{llll}
\hline & All SPs & SPs I-39a & SPs 40-78b \\
\hline SPs with predicted TMH/Total number of SPs & $62 / 78$ & $38 / 39$ & $24 / 39$ \\
SPs without predicted TMH (\%) & 21 & 2.6 & 38 \\
\hline
\end{tabular}

aThe best performing half of the SPs

bThe least performing half of the SPs 
Table 4: Correlation between the presence of anchoring motifs ${ }^{a}(A M)$ in the natural cognate protein and measured extracellular NucA activities.

\begin{tabular}{llll}
\hline & All SPs & SPs I-39b & SPs 40-78c \\
\hline SPs with AM/Total number of SPs & $41 / 78$ & $16 / 39$ & $25 / 39$ \\
SPs without AM (\%) & 47 & 59 & 36 \\
\hline
\end{tabular}

alnvestigated anchoring motifs: LPxTG, LysM domain and C-terminal

transmembrane domain, as annotated by the Secretome

database of L. plantarum [ I8] http://www.cmbi.ru.nl/secretome.

bThe best performing half of the SPs

cThe least performing half of the SPs

Tris gels using MOPS as running buffer (both Invitrogen). Proteins were visualized using the SilverSNAP Stain for Mass Spectrometry kit from Pierce (Rockford, IL, USA) for extracellular proteins and Coomassie Brilliant Blue for intracellular proteins.

\section{Western blot analysis}

For Western blotting $2 \mathrm{ml}$ cell cultures were handled essentially as described by Piard et al.[61]. The proteins from the supernatant were precipitated by adding $400 \mu \mathrm{l}$ ice-cold $80 \%(\mathrm{v} / \mathrm{v})$ trichloroacetic acid (TCA) to $1.6 \mathrm{ml}$ supernatant. The solution was incubated on ice for $30 \mathrm{~min}$ and the resulting precipitate was collected by centrifugation at $4{ }^{\circ} \mathrm{C}$ for $10 \mathrm{~min}$ at $16000 \times \mathrm{g}$. The precipitate was washed with $300 \mu \mathrm{l}$ ice-cold acetone and recentrifuged. After freeze drying, the protein pellet was dissolved in 25 $\mu \mathrm{l}$ NuPAGE LDS sample Buffer, $10 \mu \mathrm{l}$ NuPAGE Reducing Agent (both Invitrogen) and $65 \mu \mathrm{l} 10 \mathrm{mM}$ Tris-HCl buffer (pH 8).

To extract intracellular protein, the cell pellets were washed once with TES-buffer (25\% w/v sucrose, $1 \mathrm{mM}$ EDTA and $50 \mathrm{mM}$ Tris- $\mathrm{HCl}, \mathrm{pH} 5.8$ ). The cell wall was then partially digested by adding $500 \mu \mathrm{l}$ TES buffer containing lysozyme $(16 \mathrm{mg} / \mathrm{ml})$, mutanolysin $(60 \mathrm{U} / \mathrm{ml})$ and RNase $(0.5 \mathrm{mg} / \mathrm{ml})$ (all from Sigma-Aldrich Inc, St. Louis, MO). After incubating the cell suspension for 1 hour at $37^{\circ} \mathrm{C}$, protoplasts were collected by centrifugation at $15000 \times \mathrm{g}$ for $3 \mathrm{~min}$. The protoplasts were then lysed with $85.5 \mu \mathrm{l}$ TES buffer containing, $12,5 \mu \mathrm{l} 10 \%(\mathrm{w} / \mathrm{v})$ sodium dodecyl sulphate (SDS) and the solution volume was adjusted to $125 \mu \mathrm{l}$ with $20 \mu \mathrm{l}$ NuPAGE Loading buffer (Invitrogen) and $6 \mu \mathrm{l}$ NuPAGE Reducing agent (Invitrogen). Samples were denatured at $100^{\circ} \mathrm{C}$ for 10 minutes.

One microliter samples were run on 10\% NuPAGE Novex Bis Tris Gels (Invitrogen) using MES (Invitrogen) as running buffer. Electroblotting was performed by using the iBlot Dry Blotting System (Invitrogen) according to manufacturer's recommendations, with the exception of the nitrocellulose membrane being replaced by a PVDF membrane (BioRad Laboratories, Inc, Hercules, CA). Rabbit polyclonal anti-NucA antiserum against the peptide EFD-
KGQRTDKYGRG [62] was obtained from ProSci Inc. (Poway, CA) and used as recommended by the manufacturer. Immunodetection was performed using a horseradish peroxidase-conjugated (HRP) goat anti-rabbit antibody (Bio-Rad) and the enhanced chemiluminescent kit from Pierce (Rockford, Il).

\section{Quantitative real-time PCR}

Total RNA was isolated from cell cultures harvested at $\mathrm{OD}_{600} \sim 1.7$ using the RNeasy Mini Kit (QIAGEN) with oncolumn digestion of DNA with RNase-Free DNase Set (QIAGEN). After harvesting, cell pellets (from 0.5 or $1 \mathrm{ml}$ of culture) were resuspended in $350 \mu$ l RLT buffer (RNeasy Mini Kit) containing $0.1 \%(\mathrm{v} / \mathrm{v}) \beta$-mercaptoethanol (Sigma). Cells were directly transferred to FastPrep tubes (MP Biomedicals) containing glass beads $(\leq 106$ micron, Sigma) and $300 \mu \mathrm{l}$ chloroform, and subsequently disrupted using a FastPrep-24 instrument (MP Biomedicals). After a short centrifugation, the water-phases from each sample were transferred to a new RNase free tube and centrifuged at $16000 \times \mathrm{g}$ for $2 \mathrm{~min}$. The supernatant was mixed with $250 \mu \mathrm{l}$ ethanol and subsequently added to an RNeasy spin column. Further steps were performed according to the procedure of the RNeasy Mini Kit (QIAGEN). After RNA isolation, an additional DNase treatment was performed using TURBO DNase (Applied Biosystems, Foster City, CA) following the manufacturer's instructions. RNA concentrations were quantified using the NanoDrop spectrophotometer (Thermo Fisher Scientific Inc, Waltham, MA) and the quality of the RNA was assessed using the RNA 600 Nano LabChip kit and the Bioanalyzer 2100 (Agilent Technologies, Inc, Santa Clara CA). Control of residual chromosomal DNA from the total RNA isolation was performed on DNase treated samples. RNA was isolated from two independent cultures of each transformant, and these were analyzed as independent replicates throughout the real-time PCR procedure.

Synthesis of cDNA was performed using the Superscript III kit (Invitrogen) according to the manufacturer's instructions. Five-hundred nanogram total RNA and 100 ng random primers (Invitrogen) were used in each reaction. 
All real-time PCR amplifications were performed using a 7900 HT Fast Real-Time PCR system (Applied Biosystems) with standard block, and data were analyzed using the Sequence Detection Systems software (Applied Biosystems).

PCR efficiencies (E) for the primer pairs for $n u c A$ and the reference gene gyrA (see additional file 4) were calculated from the slope of standard curves consisting of the amplification results from five 10-fold dilutions of a pool of

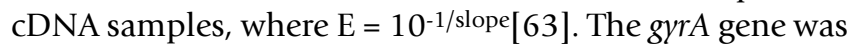
chosen as reference gene because it is known to be constitutively and stably expressed under various conditions in lactic acid bacteria [64]. The PCR program consisted of an initial denaturation step at $95^{\circ} \mathrm{C}$ for $10 \mathrm{~min}$., followed by 40 cycles of $95^{\circ} \mathrm{C}$ for $15 \mathrm{~s}$ and $60^{\circ} \mathrm{C}$ for $1 \mathrm{~min}$. Amplification was followed by melting curve analysis and determination of melting temperature for the PCR products, as a control of amplification specificity. Each PCR reaction contained $400 \mathrm{nM}$ of gene-specific primers and $2 \mu \mathrm{l}$ diluted $(25 \times)$ cDNA in a total volume of $25 \mu \mathrm{l}$ SYBR Green PCR Master Mix (Applied Biosystems). All reactions were assessed in triplicate. Relative expression of nucA is based on the ratio of the nucA transcript versus the reference gene transcript (gyrA), in cultures with cells containing the specific $n u c A$ construct, and was calculated using the relative expression software tool (REST) [65]. A randomly selected nucA construct (pLp_3093sNuc) was used as the control for all other samples in REST calculations, and expression ratios were calculated accordingly. The expression ratio results were tested for significance by a pair wise fixed reallocation test using REST [65].

\section{Scanning electron microscopy}

Cells were harvested at $\mathrm{OD}_{600} \sim 1.7$ by centrifugation at $2000 \times \mathrm{g}$ for $3 \mathrm{~min}$ and subsequently washed with $2 \mathrm{ml}$ $0.9 \%(\mathrm{w} / \mathrm{v}) \mathrm{NaCl}$. The suspensions were centrifuged and the resulting pellets were stored at $-20^{\circ} \mathrm{C}$ until use. Immediately prior to the analyses, cells were thawed on ice for $20 \mathrm{~min}$ and suspended in $1 \mathrm{ml} 0.1 \mathrm{M}$ Tris- $\mathrm{HCl}$ (pH 7.5). For scanning electron microscopy, several drops of cell suspension were transferred to glass cover slips coated with poly-L-Lysine. The cover slips were washed twice in $0.1 \mathrm{M}$ Tris-HCl (pH 7.5) to remove excess of cells. Dehydration was performed by immersing the slides in a series of ethanol solutions $(70,90,96$, and 4 times in $100 \%$ ethanol). The cover slips were placed in a critical point drier (CPD 030, Bal-Tec, Balzers, Lichtenstein), mounted on Alstubs using double faced carbon tabs (Agar Scientific, Essex, England), and subsequently coated with approximately $500 \AA$ Pt in a SC7640 sputter coater (Quorum Technologies Ltd, Newhaven, U.K.)). The dried bacteria were analyzed in a Zeiss EVO-50 (Zeiss, Jena, Germany) scanning electron instrument at $10 \mathrm{kV}$.

\section{Analysis of signal peptides}

Signal peptide cleavage sites were predicted using the SignalP 3.0 server $[31,32]$, which is accessible at http:// www.cbs.dtu.dk/services/SignalP/. The Secretome database of $L$. plantarum WCFS1 was assessed at http:// www.cmbi.ru.nl/secretome[18]. LocateP was assessed at http://www.cmbi.ru.nl/locatep-db/cgi-bin/locatepdb.py.

[54]. Transmembrane predictions of the signal sequences were performed using the TMHMM Server v. 2.0 [40], which is accessible at http://www.cbs.dtu.dk/services/ TMHMM-2.0/. The hydrophobicity of the SPs was estimated using the ProtScale program [66] and the Kyte \& Doolittle scale [67] on the ExPASy Server http:// ca.expasy.org/tools/protscale.html, using a sliding window of seven residues. Composition maps were made using the WebLogo application [34] which is accessible at http://weblogo.berkeley.edu/.

\section{Authors' contributions}

GM, LA and VE developed the initial concept for this study. GM, LA and VE participated in experimental design and coordination of the study. AS and GM constructed the library, carried out the enzyme assays, western analysis and analyzed the signal peptides. GM and MBB carried out the real-time PCR analysis and LF carried out the scanning electron microscopy. GM made the WebLogos. GM and VE drafted the paper, implementing contributions from all other authors. All authors read, corrected and approved the final manuscript.

\section{Additional material}

\section{Additional file 1}

List of signal peptides. Properties of the signal peptides (SPs) used in the present study and NucA activity in the supernatants of cells harbouring SP-NucA constructs.

Click here for file

[http://www.biomedcentral.com/content/supplementary/14712164-10-425-S1.pdf]

\section{Additional file 2}

WebLogos of signal peptides. Frequency plots made with WebLogo [34], based on multiple alignment of the sequence starting13 residues upstream of the predicated cleavage site and ending two residues downstream. Click here for file

[http://www.biomedcentral.com/content/supplementary/14712164-10-425-S2.pdf]

\section{Additional file 3}

SDS-PAGE analysis of $\mathrm{L}$. plantarum containing various constructs for amylase secretion. Coomassie stained and Silver-stained SDS-PAGE gels of cell extracts and cell free supernatants of L. plantarum WCFS1 harbouring various amylase constructs.

Click here for file

[http://www.biomedcentral.com/content/supplementary/14712164-10-425-S3.pdf] 


\section{Additional file 4}

List of primers. Primers used in real-time PCR experiments and for PCRamplification of signal peptides from genomic DNA of L. plantarum WCFS1.

Click here for file

[http://www.biomedcentral.com/content/supplementary/1471-

2164-10-425-S4.pdf]

\section{Acknowledgements}

This work was supported by grant 159058 from the Research Council of Norway. We are grateful to Trygve Krekling for excellent assistance with the scanning electron microscopy experiments and we thank Sigrid Gåseidnes for fruitful discussions.

\section{References}

I. Ahrné S, Nobaek S, Jeppsson B, Adlerberth I, Wold AE, Molin G: The normal Lactobacillus flora of healthy human rectal and oral mucosa. J Appl Microbiol 1998, 85:88-94.

2. Kleerebezem M, Boekhorst J, van Kranenburg R, Molenaar D, Kuipers OP, Leer R, Tarchini R, Peters SA, Sandbrink HM, Fiers MWEJ, et al.: Complete genome sequence of Lactobacillus plantarum WCFSI. Proc Natl Acad Sci USA 2003, I00:1990-1995.

3. Sørvig E, Mathiesen G, Naterstad K, Eijsink VGH, Axelsson L: Highlevel, inducible gene expression in Lactobacillus sakei and Lactobacillus plantarum using versatile expression vectors. Microbiology 2005, I 5 I :2439-2449.

4. Hols P, Ferain T, Garmyn D, Bernard N, Delcour J: Use of homologous expression-secretion signals and vector-free stable chromosomal integration in engineering of Lactobacillus plantarum for $\alpha$-amylase and levanase expression. Appl Environ Microbiol 1994, 60:|40|-|4|3.

5. Hols P, Slos P, Dutot P, Reymund J, Chabot P, Delplace B, Delcour J, Mercenier A: Efficient secretion of the model antigen M6gp4IE in Lactobacillus plantarum NCIMB 8826. Microbiology 1997, | 43:2733-274I.

6. Pavan S, Hols P, Delcour J, Geoffroy MC, Grangette C, Kleerebezem $M$, Mercenier A: Adaptation of the nisin-controlled expression system in Lactobacillus plantarum: a tool to study in vivo biological effects. Appl Environ Microbiol 2000, 66:4427-4432.

7. Lambert JM, Bongers RS, Kleerebezem M: Cre-lox-based system for multiple gene deletions and selectable-marker removal in Lactobacillus plantarum. Appl Environ Microbiol 2007, 73: II26-II35.

8. Bron PA, Grangette C, Mercenier A, de Vos WM, Kleerebezem M: Identification of Lactobacillus plantarum genes that are induced in the gastrointestinal tract of mice. J Bacteriol 2004, I 86:572I-5729.

9. Marco ML, Bongers RS, de Vos WM, Kleerebezem M: Spatial and temporal expression of Lactobacillus plantarum genes in the gastrointestinal tracts of mice. Appl Environ Microbiol 2007, 73: $124-132$.

10. de Vries MC, Vaughan EE, Kleerebezem M, de Vos WM: Lactobacillus plantarum - survival, functional and potential probiotic properties in the human intestinal tract. International Dairy Journal 2006, 16:1018-1028.

II. Braat H, Rottiers P, Hommes DW, Huyghebaert N, Remaut E, Remon JP, van Deventer SJ, Neirynck S, Peppelenbosch MP, Steidler L: A phase I trial with transgenic bacteria expressing interleukinI 0 in Crohn's disease. Clin Gastroenterol Hepatol 2006, 4:754-759.

12. Wells JM, Mercenier A: Mucosal delivery of therapeutic and prophylactic molecules using lactic acid bacteria. Nat Rev Microbiol 2008, 6:349-362.

13. Mohamadzadeh M, Duong T, Sandwick SJ, Hoover T, Klaenhammer TR: Dendritic cell targeting of Bacillus anthracis protective antigen expressed by Lactobacillus acidophilus protects mice from lethal challenge. Proc Natl Acad Sci USA 2009, I 06:4331-4336.
14. Papanikou E, Karamanou S, Economou A: Bacterial protein secretion through the translocase nanomachine. Nat Rev Microbiol 2007, 5:839-85I.

I5. Tjalsma H, Antelmann H, Jongbloed JD, Braun PG, Darmon E, Dorenbos R, Dubois JY, Westers H, Zanen G, Quax WJ, et al.: Proteomics of protein secretion by Bacillus subtilis: separating the "secrets" of the secretome. Microbiol Mol Biol Rev 2004, 68:207-233.

16. van Wely KHM, Swaving J, Freudl R, Driessen AJM: Translocation of proteins across the cell envelope of Gram-positive bacteria. FEMS Microbiol Rev 200I, 25:437-454.

17. von Heijne G: The signal peptide. J Membr Biol I990, I I 5: I 95-20I.

18. Boekhorst J, Wels M, Kleerebezem M, Siezen RJ: The predicted secretome of Lactobacillus plantarum WCFSI sheds light on interactions with its environment. Microbiology 2006, I 52:3175-3183.

19. Mathiesen G, Sveen A, Piard JC, Axelsson L, Eijsink VGH: Heterologous protein secretion by Lactobacillus plantarum using homologous signal peptides. J Appl Microbiol 2008, 1 05:2 I 5-226.

20. Savijoki K, Kahala M, Palva A: High level heterologous protein production in Lactococcus and Lactobacillus using a new secretion system based on the Lactobacillus brevis S-layer signals. Gene 1997, I 86:255-262.

21. Le Loir Y, Nouaille S, Commissaire J, Bretigny L, Gruss A, Langella P: Signal peptide and propeptide optimization for heterologous protein secretion in Lactococcus lactis. Appl Environ Microbiol 2001, 67:4I 19-4I27.

22. Hazebrouck S, Oozeer R, Adel-Patient K, Langella P, Rabot S, Wal JM, Corthier G: Constitutive delivery of bovine $\beta$-lactoglobulin to the digestive tracts of gnotobiotic mice by engineered Lactobacillus casei. Appl Environ Microbiol 2006, 72:7460-7467.

23. Nijland R, Lindner C, van Hartskamp M, Hamoen LW, Kuipers OP: Heterologous production and secretion of Clostridium perfringens beta-toxoid in closely related Gram-positive hosts. J Biotechnol 2007, I 27:36I-372.

24. Oh Y, Varmanen P, Han XY, Bennett G, Xu Z, Lu T, Palva A: Lactobacillus plantarum for oral peptide delivery. Oral Microbiol Immunol 2007, 22:140-I44.

25. Steidler L, Neirynck S, Huyghebaert N, Snoeck V, Vermeire A, Goddeeris B, Cox E, Remon JP, Remaut E: Biological containment of genetically modified Lactococcus lactis for intestinal delivery of human interleukin I O. Nat Biotechnol 2003, 2 I:785-789.

26. Dieye Y, Usai S, Clier F, Gruss A, Piard JC: Design of a protein-targeting system for lactic acid bacteria. J Bacteriol 200I, | 83:4|57-4166.

27. Slos P, Dutot P, Reymund J, Kleinpeter P, Prozzi D, Kieny MP, Delcour J, Mercenier A, Hols P: Production of cholera toxin B subunit in Lactobacillus. FEMS Microbiol Lett 1998, 169:29-36.

28. Cortes-Perez NG, Azevedo V, Alcocer-Gonzalez JM, RodriguezPadilla C, Tamez-Guerra RS, Corthier G, Gruss A, Langella P, Bermudez-Humaran LG: Cell-surface display of E7 antigen from human papillomavirus type- 16 in Lactococcus lactis and in Lactobacillus plantarum using a new cell-wall anchor from lactobacilli. J Drug Target 2005, I 3:89-98.

29. Reveneau N, Geoffroy MC, Locht C, Chagnaud P, Mercenier A: Comparison of the immune responses induced by local immunizations with recombinant Lactobacillus plantarum producing tetanus toxin fragment $\mathbf{C}$ in different cellular locations. Vaccine 2002, 20:I769-I777.

30. Kerovuo J, Tynkkynen S: Expression of Bacillus subtilis phytase in Lactobacillus plantarum 755. Lett Appl Microbiol 2000, 30:325-329.

3I. Bendtsen JD, Nielsen H, von Heijne G, Brunak S: Improved prediction of signal peptides: SignalP 3.0. J Mol Biol 2004, 340:783-795.

32. Nielsen H, Engelbrecht J, Brunak S, von Heijne G: Identification of prokaryotic and eukaryotic signal peptides and prediction of their cleavage sites. Protein Eng 1997, I0:1-6.

33. Antelmann H, Tjalsma H, Voigt B, Ohlmeier S, Bron S, van Dijl JM, Hecker M: A proteomic view on genome-based signal peptide predictions. Genome Res 2001, I I:I484-I502.

34. Crooks GE, Hon G, Chandonia JM, Brenner SE: WebLogo: a sequence logo generator. Genome Res 2004, I 4: I I 88-I 190.

35. Le Loir Y, Gruss A, Ehrlich SD, Langella P: A nine-residue synthetic propeptide enhances secretion efficiency of heterologous proteins in Lactococcus lactis. J Bacteriol 1998, 180:1895-1903. 
36. Gold RS, Meagher MM, Hutkins R, Conway T: Ethanol tolerance and carbohydrate-metabolism in Lactobacilli. J Ind Microbiol 1992, 10:45-54.

37. Giraud E, Cuny G: Molecular characterization of the alphaamylase genes of Lactobacillus plantarum A6 and Lactobacillus amylovorus reveals an unusual 3 ' end structure with direct tandem repeats and suggests a common evolutionary origin. Gene 1997, 198:149-157.

38. Wang Z, Jones JD, Rizo J, Gierasch LM: Membrane-bound conformation of a signal peptide: a transferred nuclear Overhauser effect analysis. Biochemistry (Mosc) 1993, 32:13991-13999.

39. Briggs MS, Cornell DG, Dluhy RA, Gierasch LM: Conformations of signal peptides induced by lipids suggest initial steps in protein export. Science 1986, 233:206-208.

40. Krogh A, Larsson B, von Heijne G, Sonnhammer EL: Predicting transmembrane protein topology with a hidden Markov model: application to complete genomes. J Mol Biol 200I, 305:567-580.

4I. Bolhuis A, Tjalsma H, Smith HE, de Jong A, Meima R, Venema G, Bron $S$, van Dijl JM: Evaluation of bottlenecks in the late stages of protein secretion in Bacillus subtilis. Appl Environ Microbiol 1999, 65:2934-294I

42. Brockmeier U, Caspers M, Freudl R, Jockwer A, Noll T, Eggert T: Systematic screening of all signal peptides from Bacillus subtilis: a powerful strategy in optimizing heterologous protein secretion in Gram-positive bacteria. I Mol Biol 2006, 362:393-402.

43. Stephenson K, Jensen CL, Jørgensen ST, Lakey JH, Harwood CR: The influence of secretory-protein charge on late stages of secretion from the Gram-positive bacterium Bacillus subtilis. Biochem J 2000, 350:31-39.

44. Harwood CR, Cranenburgh R: Bacillus protein secretion: an unfolding story. Trends Microbiol 2008, I 6:73-79.

45. Zanen G, Antelmann H, Meima R, Jongbloed JD, Kolkman M, Hecker $M$, van Dijl JM, Quax WJ: Proteomic dissection of potential signal recognition particle dependence in protein secretion by Bacillus subtilis. Proteomics 2006, 6:3636-3648.

46. Fu LL, Xu ZR, Shuai JB, Hu CX, Dai W, Li WF: High-level secretion of a chimeric thermostable lichenase from Bacillus subtilis by screening of site-mutated signal peptides with structural alterations. Curr Microbiol 2008, 56:287-292.

47. Zanen G, Houben ENG, Meima R, Tjalsma H, Jongbloed JDH, Westers H, Oudega B, Luirink J, van Dijl JM, Quax WJ: Signal peptide hydrophobicity is critical for early stages in protein export by Bacillus subtilis. FEBS Journal 2005, 272:46I7-4630.

48. Ravn P, Arnau J, Madsen SM, Vrang A, Israelsen H: Optimization of signal peptide SP3 I 0 for heterologous protein production in Lactococcus lactis. Microbiology 2003, I 49:2 I 93-220 I.

49. Carlsson F, Stalhammar-Carlemalm M, Flardh K, Sandin C, Carlemalm E, Lindahl G: Signal sequence directs localized secretion of bacterial surface proteins. Nature 2006, 442:943-946.

50. DeDent A, Bae T, Missiakas DM, Schneewind O: Signal peptides direct surface proteins to two distinct envelope locations of Staphylococcus aureus. EMBO J 2008, 27:2656-2668.

51. Hegde RS, Bernstein HD: The surprising complexity of signal sequences. Trends Biochem Sci 2006, 3 I:563-57I.

52. Borchert TV, Nagarajan V: Effect of signal sequence alterations on export of levansucrase in Bacillus subtilis. J Bacteriol I991, I 73:276-282.

53. Hikita C, Mizushima S: Effects of total hydrophobicity and length of the hydrophobic domain of a signal peptide on in vitro translocation efficiency. J Biol Chem 1992, 267:4882-4888.

54. Zhou M, Boekhorst J, Francke C, Siezen RJ: LocateP: genomescale subcellular-location predictor for bacterial proteins. BMC Bioinformatics 2008, 9:173.

55. Hyyryläinen H-L, Bolhuis A, Darmon E, Muukkonen L, Koski $P$, Vitikainen M, Sarvas M, Prágai Z, Bron S, Dijl JMv, et al.: A novel twocomponent regulatory system in Bacillus subtilis for the survival of severe secretion stress. Mol Microbiol 2001, 4I:II59-II72.

56. Lulko AT, Veening J-W, Buist G, Smits WK, Blom EJ, Beekman AC, Bron S, Kuipers OP: Production and secretion stress caused by overexpression of heterologous $\alpha$-Amylase leads to inhibition of sporulation and a prolonged motile phase in Bacillus subtilis. Appl Environ Microbiol 2007, 73:5354-5362.
57. Aukrust TW, Brurberg MB, Nes IF: Transformation of Lactobacillus by electroporation. Methods Mol Biol 1995, 47:201-208.

58. Sørvig E, Grönqvist S, Naterstad K, Mathiesen G, Eijsink VGH, Axelsson L: Construction of vectors for inducible gene expression in Lactobacillus sakei and L. plantarum. FEMS Microbiol Lett 2003, 229: $119-126$

59. Eijsink VGH, Brurberg MB, Middelhoven PH, Nes IF: Induction of bacteriocin production in Lactobacillus sake by a secreted peptide. J Bacteriol 1996, I 78:2232-2237.

60. Heins JN, Taniuchi H, Anfinsen CB: Extracellular nuclease from Staphylococcus aureus. In Procedures in nucleic acid research Edited by: Cantoni GL, David R. New York: Harper \& Row; 1966:79-84.

61. Piard JC, Hautefort I, Fischetti VA, Ehrlich SD, Fons M, Gruss A: Cell wall anchoring of the Streptococcus pyogenes M6 protein in various lactic acid bacteria. J Bacteriol 1997, I 79:3068-3072.

62. MacConaill LE, Fitzgerald GF, van Sinderen D: Investigation of protein export in Bifidobacterium breve UCC2003. Appl Environ Microbiol 2003, 69:6994-700I.

63. Pfaffl MW: A new mathematical model for relative quantification in real-time RT-PCR. Nucleic Acids Res 2001, 29:e45.

64. Desroche N, Beltramo C, Guzzo J: Determination of an internal control to apply reverse transcription quantitative PCR to study stress response in the lactic acid bacterium Oenococcus oeni. J Microbiol Methods 2005, 60:325-333.

65. Pfaffl MW, Horgan GW, Dempfle L: Relative expression software tool (REST) for group-wise comparison and statistical analysis of relative expression results in real-time PCR. Nucleic Acids Res 2002, 30:e36.

66. Gasteiger E, Hoogland C, Gattiker A, Duvaud S, Wilkins MR, Appel $R D, A B$ : Protein identification and analysis tools on the ExPASy server. In The Proteomics Protocols Handbook Edited by: M WJ. Humana Press; 2005:57I-607.

67. Kyte J, Doolittle RF: A simple method for displaying the hydropathic character of a protein. J Mol Biol 1982, I 57:105-132.
Publish with Biomed Central and every scientist can read your work free of charge

"BioMed Central will be the most significant development for disseminating the results of biomedical research in our lifetime. "

Sir Paul Nurse, Cancer Research UK

Your research papers will be:

- available free of charge to the entire biomedical community

- peer reviewed and published immediately upon acceptance

- cited in PubMed and archived on PubMed Central

- yours - you keep the copyright
BioMedcentral 